EPJ manuscript No.

(will be inserted by the editor)

\title{
Nucleon sigma term and quark condensate in nuclear matter
}

\author{
K. Tsushima ${ }^{1}$, K. Saito ${ }^{2}$, A. W. Thomas ${ }^{3}$ and A. Valcarce ${ }^{1}$
}

1 Universidad de Salamanca, E-37008, Salamanca, Spain

2 Tokyo University of Science, Noda 278-8510, Japan

Jefferson Lab, 12000 Jefferson Avenue, Newport News, VA 23606, U.S.A.

Received: date / Revised version: date

\begin{abstract}
We study the bound nucleon sigma term and its effect on the quark condensate in nuclear matter. In the quark-meson coupling (QMC) model it is shown that the nuclear correction to the sigma term is small and negative. Thus, the correction decelerates the decrease of the quark condensate in nuclear matter. However, the quark condensate in nuclear matter is controlled primarily by the scalar-isoscalar $\sigma$ field of the model. It appreciably moderates the decrease relative to the leading term at densities around and larger than the normal nuclear matter density.
\end{abstract}

PACS. $24.85+$ p Quarks in Nuclei - 11.30.Rd Chiral symmetries - 21.65.+f Nuclear matter

\section{Introduction}

One of the most challenging questions in nuclear physics is whether or not the properties of nucleon and hadrons are modified in nuclei [1]2]. A number of pieces of evidence, such as the nuclear EMC effect 3, the quenching [4,5, U6 (enhancing 7]) of the space (time) component of the axial coupling constant in nuclear $\beta$ decays, the missing strength of the response functions and the suppression of the Coulomb sum rule [8, have stimulated investigations of this question. In particular, to focus on the change of the internal structure of the nucleon (hadrons) is very appealing in the light of an approach based on QCD.

In addition to the recent experimental evidence for the vector meson mass shifts in nuclei [9], some important hints concerning the change of the internal structure of a bound nucleon has been reported in measurements of the electromagnetic form factors of a proton in ${ }^{4} \mathrm{He}$ [11. The analyses suggest a reduction of the bound proton's electric to magnetic form factor ratio, where most of the conventional, sophisticated approaches employing the free proton form factors [12] fail to explain the observed effect. (See Ref. [13] for an exploration of a conventional mechanism which might explain at least some of the effect. We note, however, that for the moment that approach is hampered by a lack of experimental data to pin down the input parameters.) Indeed, excellent agreement with the data is achieved when the small correction associated with the change of the internal structure of the bound proton (which had been predicted 14] long before the experiments) is included.

Up to now, some models such as the quark-meson coupling (QMC) model [15]16]17] and Nambu-Jona-Lasinio model [18, have opened possibilities to understand the change of the nucleon internal structure in nuclei based on quark degrees of freedom. However, it is important to explore other ways to study the change in the internal structure of the bound nucleon through various nuclear phenomena.

In this note, we study the bound nucleon sigma term, which has a direct connection with the QCD Hamiltonian via Feynman-Hellmann theorem [19]. First, we study the density dependence of the nuclear correction to the sigma term in the QMC model [15]16]. Next, we study the influence of the nuclear correction that arises from the sigma term on the quark condensate.

\section{Bound nucleon sigma term}

For the ground state of uniform, nucleon density $\rho_{B}$ with A nucleons and volume $V\left(\rho_{B}=A / V\right)$, the nuclear sigma term $\sigma_{A}$ and the bound nucleon sigma term $\sigma_{N}^{*}$ may be defined by 20,21,22,

$$
\begin{aligned}
\sigma_{A} & \equiv A \sigma_{N}^{*}\left(\rho_{B}\right), \\
& =\frac{1}{3} \sum_{a=1}^{3}\left[<A\left(\rho_{B}\right)\left|\left[Q_{5}^{a},\left[Q_{5}^{a}, H_{Q C D}\right]\right]\right| A\left(\rho_{B}\right)>\right. \\
& \left.\quad-<0\left|\left[Q_{5}^{a},\left[Q_{5}^{a}, H_{Q C D}\right]\right]\right| 0>\right], \quad(1) \\
& =V \rho_{B} 2 m_{q}\left[<A\left(\rho_{B}\right)|\bar{q} q| A\left(\rho_{B}\right)>-<0|\bar{q} q| 0>\right],(2)
\end{aligned}
$$

where $Q_{5}^{a}$ is the weak axial charge with isospin $a$, and $H_{Q C D}$ the QCD Hamiltonian with the current quark mass, $m_{q} \equiv \frac{1}{2}\left(m_{u}+m_{d}\right)$, and $\bar{q} q \equiv \frac{1}{2}(\bar{u} u+\bar{d} d)$. Applying the Feynman-Hellmann theorem [19], we get:

$$
A \sigma_{N}^{*}\left(\rho_{B}\right)=m_{q} \frac{\partial}{\partial m_{q}}\left[<A\left(\rho_{B}\right)\left|H_{Q C D}\right| A\left(\rho_{B}\right)>\right.
$$




$$
\begin{aligned}
&\left.-<0\left|H_{Q C D}\right| 0>\right], \\
&=V \rho_{B} m_{q} \frac{\partial}{\partial m_{q}}\left[m_{N}\left(m_{q}\right)+\varepsilon_{B}\left(m_{q}, \rho_{B}\right)\right], \\
& \equiv A\left[\sigma_{N \text { free }}+\delta \sigma_{N}^{*}\left(\rho_{B}\right)\right],
\end{aligned}
$$

where $\sigma_{N \text { free }} \equiv m_{q} \frac{\partial m_{N}}{\partial m_{q}}$ is the free nucleon sigma term (empirical value $\simeq 45 \mathrm{MeV}[\underline{23}]), \delta \sigma_{N}^{*}\left(\rho_{B}\right) \equiv m_{q} \frac{\partial \varepsilon_{B}\left(m_{q}, \rho_{B}\right)}{\partial m_{q}}$ the nuclear correction to the sigma term, and $\varepsilon_{B}\left(m_{q}, \rho_{B}\right)$ the binding energy per nucleon. At present, $\varepsilon_{B}\left(m_{q}, \rho_{B}\right)$ which contains the contributions from the nucleon kinetic energy and N-N interaction etc., can only be calculated in a model dependent way. Note that our focus concerns the dependence on the current quark mass $m_{q}$ [19], which has a direct connection with the QCD Hamiltonian through Eqs. (2)-(5). It may be contrasted with the calculation of the quark condensate in nuclear matter [24,25].26.27, which will also be studied later. For later convenience we recall the saturation conditions for nuclear matter:

$$
\begin{aligned}
\left.\frac{\partial \varepsilon_{B}\left(m_{q}, \rho_{B}\right)}{\partial \rho_{B}}\right|_{\rho_{B}=\rho_{0}} & =0, \\
\varepsilon_{B}\left(m_{q}, \rho_{B}=\rho_{0}\right) & =-15.7 \mathrm{MeV} .
\end{aligned}
$$

Now we study the density dependence of the nuclear correction to the nucleon sigma term $\delta \sigma_{N}^{*}\left(\rho_{B}\right)$ in Eq. (5). Generally, models for nuclear matter based on quark degrees of freedom contain coupling constants, $g_{j}\left(m_{q}\right)(j=$ $1,2, \ldots)$, which depend on the current quark mass $m_{q}$. These coupling constants are determined so as to satisfy the saturation conditions Eqs. (6) and (7) for a chosen value of $m_{q}$. This also fixes the function $\varepsilon\left(m_{q}, \rho_{B}\right)$ as a function of $m_{q}$. Of course, since in nature we have only one set of quark masses, which lead to nuclear saturation at the correct place, it is not appropriate to include the variation of $g_{j}$ with $m_{q}$ in calculating the nuclear sigma term.

In the following we use different versions of the QMC model (QMC-I [15], and QMC-II [16] with parameter set $\mathrm{B}$ ), and study the density dependence of the nuclear correction $\delta \sigma_{N}^{*}\left(\rho_{B}\right)$. We use the standard parameters, $m_{q}=5$ $\mathrm{MeV}$, and the free nucleon bag radius $R_{N}=0.8 \mathrm{fm}$ (all the corresponding parameters, values for the coupling constants can be found in Refs. 2 15 [16]). Thus, we suppose that $m_{q}=5 \mathrm{MeV}$ is the value in the QCD Hamiltonian.

First, we show in Fig. 1 the nuclear correction to the sigma term, $\delta \sigma_{N}^{*}\left(\rho_{B}\right)$, together with the binding energy per nucleon, $\varepsilon_{B}=E / A-m_{N}$ (saturation curve) calculated in QMC-I and QMC-II. The negative contribution of the nuclear correction to the sigma term agrees with the result 22] based on the 2-body N-N interaction using the Skyrme model, although the correction is small in the present case. In Refs. 25]26], an effective density dependent sigma term was introduced. Although they estimated the $m_{q}$ dependence of the meson masses appearing in the $\mathrm{N}-\mathrm{N}$ interaction, in order to calculate the in-medium quark condensate, the calculation was not based on QCD but instead was rather qualitative. We do not attempt to make such an estimate for the variation of the masses of the meson fields.

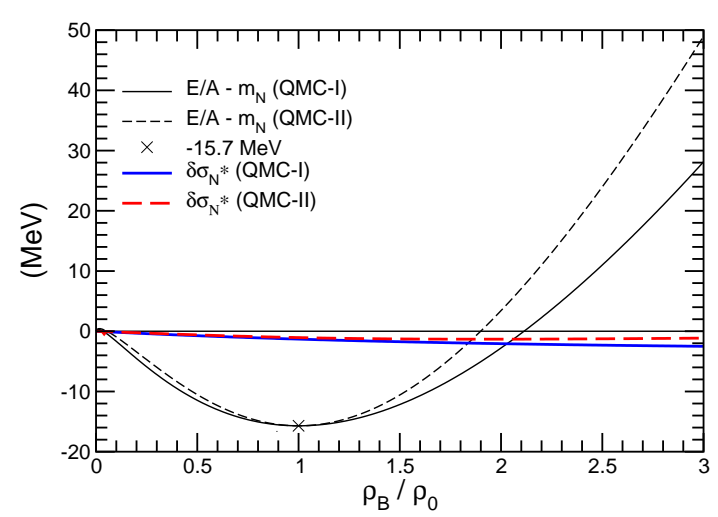

Fig. 1. Nuclear correction to the bound nucleon sigma term $\left(\delta \sigma_{N}^{*}\right)$, and the binding energy per nucleon $\left(\varepsilon_{B}=E / A-m_{N}\right)$, where $\rho_{0}=0.15 \mathrm{fm}^{-3}$.

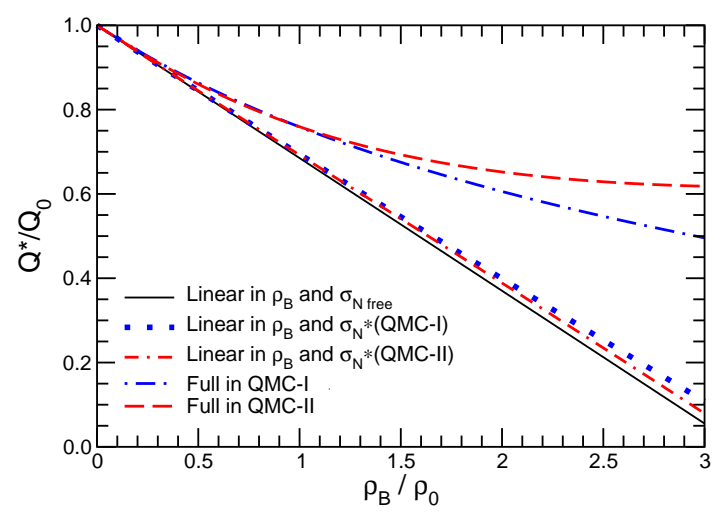

Fig. 2. The nuclear matter to vacuum quark condensate ratio. All results are obtained with $\sigma_{N \text { free }}=45 \mathrm{MeV}[23$.

\section{Quark condensate in nuclear matter}

Next, we discuss the quark condensate in nuclear matter. The quark condensate and the nuclear sigma term are related through Eqs. (31) - (5). Using the Gell-Mann-OakesRenner (GOR) relation, the nuclear matter to vacuum quark condensate ratio is given by:

$$
\frac{Q^{*}}{Q_{0}} \equiv \frac{<\rho_{B}|\bar{q} q| \rho_{B}>}{<0|\bar{q} q| 0>} \simeq 1-\frac{\sigma_{N f r e e}+\delta \sigma_{N}^{*}\left(\rho_{B}\right)}{m_{\pi}^{2} f_{\pi}^{2}} \rho_{B} .
$$

Without $\delta \sigma_{N}^{*}\left(\rho_{B}\right)$ above, it is the usual leading term result.

We show in Fig. 2 the nuclear matter to vacuum quark condensate ratio calculated via Eq. (8). The solid line is the leading term with the free nucleon sigma term, $\sigma_{N \text { free }}=45 \mathrm{MeV}$ [23], while the dotted and dash-dashdotted lines are those calculated with the bound nucleon sigma term $\sigma_{N}^{*}$ in QMC-I and QMC-II. As expected from 
the results for the bound nucleon sigma term, the influence of $\delta \sigma_{N}^{*}$ to the ratio is very small. (The full results will be discussed below.)

In the QMC model, nucleons interact through the selfconsistent exchanges of the scalar-isoscalar $(\sigma)$, vectorisoscalar $(\omega)$, and vector-isovector $(\rho)$ meson mean fields, which couple directly to the quarks inside the nucleons. In principle, although the masses of the meson fields in QMC will also depend on the current quark mass $m_{q}$, this is not a feature of the model. Thus, in the QMC model the other nuclear correction to the quark condensate, aside from the bound nucleon sigma term, is expected to arise from the scalar-isoscalar $\sigma$ field. Indeed, the full expression for the nuclear matter to vacuum quark condensate ratio in QMC-I is given by [27,

$$
\frac{Q^{*}}{Q_{0}}=1-\frac{\sigma_{N \text { free }}+\delta \sigma_{N}^{*}\left(\rho_{B}\right)}{m_{\pi}^{2} f_{\pi}^{2}}\left(\frac{m_{\sigma}}{g_{\sigma}}\right)^{2}\left(g_{\sigma} \sigma\right),
$$

where $\sigma$ is the mean value of the scalar-isoscalar $\sigma$ field in nuclear matter. For QMC-II, one may replace in Eq. (9) $m_{\sigma} \rightarrow m_{\sigma}^{*}$ which depends on density or $\sigma$ but not on $m_{q}$ explicitly. This expression, which is obtained using the self-consistent equation for the $\sigma$ field, may be a characteristic feature of the QMC model. The quark condensate in nuclear matter is directly connected to the scalarisoscalar $\sigma$ field, which plays an important role in describing the properties of nuclear matter and nuclei. This may not be a trivial result. Note that the differences between the present expression and that in Ref. 27 arise because the present one does not contain the terms involving the derivative of the quark-meson coupling constants with respect to $m_{q}$

Next, we show in Fig. 2 the full results of Eq. (9) in QMC-I (the dash-dotted line) and QMC-II (the dashed line). In both versions of QMC, the scalar-isoscalar $\sigma$ field largely moderates the decrease of the quark condensate relative to the leading term. In Refs. 25, 26, although the $m_{q}$ dependence of the exchanged mesons in the $\mathrm{N}$ $\mathrm{N}$ interaction was estimated qualitatively, they also got a similar, concave shape of the density dependence for the ratio.

\section{Summary}

To summarise, it is shown that the nuclear correction to the bound nucleon sigma term is negative, and decelerates the decrease of the quark condensate in nuclear matter. However, the magnitude is small and so is its effect on the quark condensate. The quark condensate in nuclear matter is predominantly controlled by the scalar-isoscalar $\sigma$ field in the QMC model. It moderates appreciably the decrease of the condensate relative to the leading term at densities around and larger than normal nuclear matter density.

\section{Acknowledgements}

K.T. would like to acknowledge the warm hospitality at Jefferson Lab., where part of the work was carried out.
This work was supported in part by U.S. DOE Contract No. DE-AC05-06OR23177, under which Jefferson Science Associates operate Jefferson Lab.

\section{References}

1. G. E. Brown, M. Rho, Phys. Rev. Lett. 66 (1991) 2720.

2. K. Saito, K. Tsushima and A. W. Thomas, arXiv hep-ph/0506314 to be published in Prog. Part. Nucl. Phys.

3. M. Arneodo, Phys. Rep. 240 (1994) 301; D.F. Geesaman, K. Saito, A.W. Thomas, Ann. Rev. Nucl. Part. Sci. 45 (1995) 337; R.P. Bickerstaff, A. W. Thomas, J. Phys. G 15 (1989) 1523.

4. A. Arima et al., Adv. Nucl. Phys. 18 (1987) 1; I. S. Towner, Phys. Rep. 155 (1987) 263; F. Osterfeld, Rev. Mod. Phys. 64 (1992) 491.

5. K. Tsushima, D. O. Riska, Nucl. Phys. A 549 (1992) 313.

6. D. H. Lu, A. W. Thomas, K. Tsushima, nucl-th/0112001

7. K. Kubodera, J. Delorme, M. Rho, Phys. Rev. Lett. 40 (1978) 755; M. Kirchbach, D. O. Riska, K. Tsushima, Nucl. Phys. A 542 (1992) 616; I. S. Towner, Nucl. Phys. A 542 (1992) 631.

8. J. Morgenstern, Z.-E. Meziani, Phys. Lett. B 515 (2001) 269; K. Saito, K. Tsushima, A. W. Thomas, Phys. Lett. B 465 (1999) 27.

9. D. Trnka et al. [CBELSA/TAPS Collaboration], Phys. Rev. Lett. 94 (2005) 192303.

10. M. Naruki et al., Phys. Rev. Lett. 96 (2006) 092301.

11. S. Malov et al., Phys. Rev. C 62 (2000) 057302; S. Dieterich et al., Phys. Lett. B 500 (2001) 47; S. Strauch et al., Phys. Rev. Lett. 91 (2003) 052301.

12. J. J. Kelly, Phys. Rev. C 60 (1999) 044609; J. M. Udias, J. R. Vignote, Phys. Rev. C 62 (2000) 034302; J. M. Udias et al., Phys. Rev. Lett. 83 (1999) 5451.

13. R. Schiavilla et al., Phys. Rev. Lett. 94 (2005) 072303.

14. D. H. Lu, A. W. Thomas, K. Tsushima, A. G. Williams, K. Saito, Phys. Lett. B 417 (1998) 217; D. H. Lu, K. Tsushima, A. W. Thomas, A. G. Williams, K. Saito, Phys. Rev. C 60 (1999) 068201.

15. P. A. M. Guichon, Phys. Lett. B 200 (1989) 235; P. A. M. Guichon, K. Saito, E. N. Rodionov, A. W. Thomas, Nucl. Phys. A 601 (1996) 349; K. Saito, K. Tsushima, A. W. Thomas, Nucl. Phys. A 609 (1996) 339; P. G. Blunden, G. A. Miller, Phys. Rev. C 54 (1996) 359.

16. K. Saito, K. Tsushima, A. W. Thomas, Phys. Rev. C 55 (1997) 2637.

17. P. A. M. Guichon and A. W. Thomas, Phys. Rev. Lett. 93 (2004) 132502.

18. W. Bentz and A. W. Thomas, Nucl. Phys. A 696 (2001) 138.

19. R. P. Feynman, Phys. Rev. 56 (1939) 340.

20. R. L. Jaffe, Phys. Rev. D 21 (1980) 3125.

21. J. Delome, G. Chanfray, M. Ericson, Nucl. Phys. A 603 (1996) 239.

22. A. Gammal, T. Frederico, Phys. Rev. C 57 (1998) 2830.

23. J. Gasser, H. Leutwyler, M. Sainio, Phys. Lett. B 253 (1991) 252.

24. T. D. Cohen, R. J. Furnstahl, D. K. Griegel, Phys. Rev. D 45 (1992) 1881.

25. G Q. Li, C. M. Ko, Phys. Lett. B 338 (1994) 118. 
K. Tsushima, K. Saito, A. W. Thomas, A. Valcarce: Nucleon sigma term and quark condensate in nuclear matter

26. R. Brockmann, W. Weise, Phys. Lett. B 367 (1996) 40.

27. K. Saito, K. Tsushima, A. W. Thomas, Mod. Phys. Lett. A 10 (1998) 769. 
